\title{
Diagnostic Value of Post Decontamination Smear Microscopy for Enhanced Detection of Tuberculosis
}

\author{
IJCRR \\ Section: Healthcare \\ ISI Impact Factor \\ (2020-21): 1.899 \\ IC Value (2020): 91.47 \\ SJIF $(2020)=7.893$ \\ (c) (i) (3) \\ Copyright@IJCRR
}

Swati Salila ${ }^{1}$, U. N. Gaikwad ${ }^{2}$

'Senior Resident, Department of Microbiology, All India Institute of Medical Sciences, Raipur, India; 'Additional Professor, Department of Microbiology, All India Institute of Medical Sciences, Raipur, India.

\section{ABSTRACT}

Introduction: A direct smear microscopy is a universal tool used for diagnosis of tuberculosis under programmatic settings. Early diagnosis of TB is crucial both clinically and epidemiologically. It is essential to ensure proper and early identification of cases, and good treatment outcomes to be able to limit its transmission and obtain successful TB control especially in low income countries. The study aims to evaluate the efficacy of smear microscopy performed on post-NaLC-NaOH decontaminated samples in diagnosis of Tuberculosis.

Materials and Methods: The samples were subjected to direct smear microscopy using Ziehl-Neelson (ZN) stain followed by decontamination and concentration by NALC-NaOH method and culture on Lowenstein Jensen media as well as in MGIT 960 system. Results of direct smear microscopy and post decontamination smear microscopy were compared against culture as gold standard.

Results: A total 705 samples were processed of which 658 were negative for acid fast bacilli by direct microscopy. We observed that direct microscopy showed $41.22 \%$ (47) positivity whereas microscopy by post decontamination showed $60.52 \%$ (69) positive. True diagnostic yield of $24 \%$ was obtained by post decontamination smear microscopy over direct microscopy. The overall sensitivity, specificity, PPV and NPV for direct and post decontamination smear was calculated as $36.84 \%, 99.15 \%, 89.36 \%$, $89.06 \%$ and $60.52 \%, 97.96 \%, 85.19 \%, 92.79 \%$ respectively. Thus, the sensitivity of detection was increased by $23.68 \%$ on post decontamination smear microscopy with diagnostic accuracy of $91.91 \%$.

Conclusion: Post decontamination smears showed significant increase in diagnostic yield as compared to direct smear microscopy. Performing and reporting PDSM on samples requested for culture, as an additional step, in appropriate laboratory settings can have greater impact in providing rapid and accurate diagnosis especially in smear-negative and extra-pulmonary TB cases.

Key Words: Concentration methods, Smear-negative pulmonary TB, Post decontamination smear microscopy, EPTB, Diagnostic accuracy, Sensitivity

\section{INTRODUCTION}

Direct smear microscopy is a universal tool used for diagnosis of tuberculosis(TB) under programmatic settings. Sputum smear microscopy is a century-old test but remains the primary tool for diagnosing TB in low-income countries. The Ziehl-Neelsen method has endured as a reliable and effective way to demonstrate the acid-fast bacteria. ${ }^{1}$ It is an important tool in initial screening of the disease as it can differentiate MTB which appear as long, curved and beaded bacilli from Non-Tuberculous Mycobacteria (NTM) which appear as short, straight bacilli with no specific morphology. ${ }^{2,3} \mathrm{How}-$ ever, the threshold for detection of acid-fast bacilli (AFB) in samples under optimal conditions is very high $\left(10^{4}\right.$ to $10^{5}$ bacilli/ml) making smear microscopy as a less sensitive tool for diagnosing pulmonary $\mathrm{TB} .{ }^{4}$

Although simple and cost effective, it significantly lacks sensitivity and specificity in detecting tubercle bacilli especially in extra pulmonary tuberculosis (EPTB) due to its pauci-bacillary nature of disease, the variable clinical presentations, need for invasive procedures to secure appropriate sample, and lack of laboratory facilities in the resource-limited settings ${ }^{5}$.The conventional methods of diagnosis of TB has various limitations hence rapid diagnosis of EPTB is a challenging task. Moreover, in a developing country like India

Corresponding Author:

Swati Salila, Qr. No. - E 1/3, IGIMS Campus, Sheikhpura, Patna - 800014, India.

Phone: 08521637372; Email: dr.swatisalila@gmail.com

ISSN: $2231-2196$ (Print)

ISSN: 0975-5241 (Online)

Received: 17.08 .2021

Revised: 10.10 .2021

Accepted: 13.11 .2021

Published: 15.02 .2022 
where the health care professionals are facing difficulties in diagnosing the disease due to limited infrastructure, better methods are gravely needed for control programs.

Under the TB program conditions and due to technical constraints, the yield is further decreased (IUATLD, 2005). ${ }^{6}$ Definitive and rapid diagnosis of extrapulmonary tuberculosis is challenging since conventional techniques have limitations. Smear-negative pulmonary TB (SNPT) represents 30$60 \%$ of all pulmonary TB cases, according to region. ${ }^{7}$ Early diagnosis of TB is crucial both clinically and epidemiologically. It is essential to ensure proper and early identification of cases, and good treatment outcomes to be able to limit its transmission and obtain successful TB control. In laboratory settings with culture facilities, the diagnostic capacity of smear-negative pulmonary and extrapulmonary samples can be increased by examining the post decontamination smears adding upon the rapidity of diagnosis. This is especially helpful in direct smear-negative and EPTB samples. With this hypothesis for increased diagnostic yield, the study aims to evaluate the efficacy of smear microscopy performed on post $\mathrm{NaLC}-\mathrm{NaOH}$ decontaminated samples in diagnosis of Tuberculosis.

\section{MATERIALS AND METHODS}

A cross-sectional analysis of samples requested for mycobacterial culture from clinically suspected tuberculosis cases, over a period of 18 months(Jan 2018 - Jun 2019) was done in the department of Microbiology, AIIMS, Raipur. The samples were subjected to direct smear microscopy using Ziehl-Neelson (ZN) stain followed by decontamination and concentration by NALC-NaOH method and culture on Lowenstein Jensen media as well as in MGIT 960 system. $\mathrm{ZN}$ smears were prepared from the decontaminated samples. The growth on culture was confirmed for MTB by MPT64 antigen immuno-chromatography test. Results of direct smear microscopy and post decontamination smear microscopy were compared against culture as gold standard.

\section{Data Analysis}

Results of direct smear microscopy and post decontamination smear microscopy were compared against culture as gold standard to find out: Sensitivity, Specificity, Positive Predictive Value (PPV), Negative Predictive Value (NPV), Diagnostic Accuracy. The p-value $<0.05$ considered statistically significant.

\section{RESULTS}

A total 705 samples were processed of which 237 were pulmonary and 468 were extrapulmonary. Out of these 705 samples, 658 were negative for acid-fast bacilli by direct microscopy. The positivity rate of $16 \%$ by culture (114/705) was observed in this study. We observed that direct microscopy could detect $36.84 \%$ of culture positives whereas post decontamination smear microscopy could detect $60.52 \%$ of culture positives.

Amongst these, 36 (5.47\%) comprising of 18 pulmonary and 18 extrapulmonary samples were additionally picked up as smear-positive on post decontamination smear microscopy as compared to 39 (pulmonary) and 8 (extra-pulmonary) on direct smear microscopy. Twenty-eight (77.7\%) amongst them were further confirmed as culture positive for mycobacteria. Results of direct smear and post decontamination smear microscopy was compared (Table-1)and an additional yield by post decontamination smear microscopy $=36$ $(5.47 \%)$ out of all smear negatives was observed.

Results of ZN microscopy was compared against culture as gold standard test. We observed that direct microscopy could detect $36.84 \%$ of culture positives whereas post decontamination smear microscopy could detect $60.52 \%$ of culture positives. True diagnostic yield of $24 \%$ (28/114) was obtained by post decontamination smear microscopy over direct microscopy. Amongst these 28 isolates:23 (20.17\%) were Mycobacterium tuberculosis complex, 5 (4.3\%) were nontubercular Mycobacteria, 13 (11.4\%) pulmonary samples, $15(13.15 \%)$ extrapulmonary samples (pleural fluid $>$ cervical $\mathrm{LN}>$ gastric aspirate, pus, peritoneal fluid, urine).

The overall sensitivity, specificity, PPV and NPV for direct and post decontamination smear (Table - 2) was calculated as $36.84 \%, 99.15 \%, 89.36 \%, 89.06 \%$ and $60.52 \%, 97.96 \%$, $85.19 \%, 92.79 \%$ respectively. Thus, the sensitivity of detection was increased by $23.68 \%$ on post decontamination smear microscopy with diagnostic accuracy of $91.91 \%$.

\section{DISCUSSION}

Microscopy detection rates before and after concentration of specimens were compared. The sensitivity of detection by microscopy increased by $23.68 \%$ over those without concentration. It is comparable to the study conducted by Morcillo et al. which shows increased sensitivity of concentrated microscopy by $15.2 \%$ (Hypertonic Saline-SodiumHydroxide: $73.5 \%$ ) to $16.7 \%$ (NALC-NaOH: $75.0 \%$ ) over those without concentration $(58.3 \%)^{8}$.

Ganozaet al. had conducted similar study which showed sensitivity for AFB smears was increased from $28.6 \%$ using the direct method to $71.4 \%$ (Hypertonic Saline-Sodium Hydroxide) and $66.7 \%$ (NALC-NaOH) using decontamination and concentration methods 9 .

Hence the use of the concentrated method for preparing smears for AFB microscopy increases sensitivity in identifying positive TB cases, compared to the direct method. 
There were 2 samples positive with the direct method but negative with the concentrated method one of which was found to be positive by culture. It might have occurred due to inappropriate sample concentration and smear preparation, smear preparation from a negative sample accidentally, faulty staining process, or inappropriate microscopic observation. Four specimens which were positive in both direct and concentrated smear microscopy but negative in culture. Probably these patients were taking anti-TB drugs while collecting the specimens and the dead bacilli failed to grow on L-J media. No change in Sensitivity was observed by both the methods as described $81.6 \%$ for direct smear and $82.7 \%$ for the concentrated smear in a study conducted by Barez, et al. ${ }^{10}$ In another study, Cattamanchi et al. ${ }^{11}$ failed to find a difference in sensitivity between direct and concentrated sputum smear microscopy, the calculated sensitivity of direct and concentrated smear microscopy was not significantly different ( $51 \%$ vs. 52\%).A recent study conducted by Uddin et al. showed sensitivity of direct and concentrated smear microscopy was different when using positive culture as the gold standard (71\% vs. $83 \%)^{12}$.

Limitations of this study - Effect of increased diagnostic yield by post decontamination smear microscopy on therapeutic decisions could not be assessed.

\section{CONCLUSION \& RECOMMENDATION}

Post decontamination smears showed significant increase in diagnostic yield as compared to direct smear microscopy. Performing and reporting post decontamination smear microscopy on samples requested for culture, as an additional step, in appropriate laboratory settings can have greater impact in providing rapid and accurate diagnosis especially in smear negative and extra-pulmonary TB cases.

\section{ACKNOWLEDGEMENT}

I thank Dr. U. N. Gaikwad, Addl. Prof., Deptt. of Microbiology, AIIMS Raipur for her full support in conducting this study.

\section{Source of Funding: None}

\section{Conflict of Interest: None}

Authors' Contribution: Dr. U. N. Gaikwad conceived the idea and encouraged Dr. Swati Salila to investigate on the hypothesis and supervised throughout the study. Data compilation and drafting was done by Dr. Swati Salila. Both the authors discussed the results and contributed to the final manuscript.

\section{REFERENCES}

1. Bishop P.J., Neuman G. The history of the Ziehl-Neelsen stain Tubercle, 51 (1970), pp. 196-206

2. Ritu Singhal, Vithal Prasad Myneedu. Microscopy as a diagnostic tool in pulmonary tuberculosis, Int J of Mycobacteriology, Volume 4, Issue 1, 2015, Pages 1-6, ISSN 2212-5531.

3. C. Dye, C.J. Watt, D.M. Bleed, S.M. Hosseini, M.C. Raviglione. Evolution of tuberculosis control and prospects for reducing tuberculosis incidence, prevalence, and deaths globally JAMA, 293 (22) (2005), pp. 2767-2775

4. Magalhães JLO, Lima JFC, Araújo AA, Coutinho IO, Leal NC and Almeida AMP.Microscopic detection of Mycobacterium tuberculosis in direct or processed sputum smears, Rev Soc Bras Med Trop 51(2):237-239, March-April, 2018 doi: 10.1590/00378682-0238-2017

5. Purohit M, Mustafa T. Laboratory Diagnosis of Extra-pulmonary Tuberculosis (EPTB) in Resource-constrained Setting: State of the Art, Challenges and the Need. J Clin Diagn Res. 2015;9(4):EE01-EE6. doi:10.7860/JCDR/2015/12422.5792

6. Activity Report of the International Union Against Tuberculosis and Lung Disease, Jan-Dec 2005.

7. World Health Organization. Improving the diagnosis and treatment of smear-negative pulmonary and extrapulmonary tuberculosis among adults and adolescents. Recommendations for HIV prevalent and resource-constrained settings. 2007. Available: www.who.int

8. Morcillo N, Imperiale B, Palomino JC. New simple decontamination method improves microscopic detection and culture of mycobacteria in clinical practice. Infection and Drug Resistance 2008:1 21-26

9. Ganoza CA, Ricaldi JN, Chauca J, Rojas G, Munayco C, Agapito J, et. al. Novel hypertonic saline-sodium hydroxide (HS-SH) method for decontamination and concentration of sputum samples for Mycobacterium tuberculosis microscopy and culture. Journal of Medical Microbiology (2008), 57, 1094-1098

10. Marie YC, Barez MD, Myrna T, Mendoza MD, Regina S, Celada RMTet. al. Accuracy of AFB in relation to TB culture in detection of pulmonary tuberculosis. Phil J Microbiol Infect Dis. 1995, 24 (2): 33-36.

11. Cattamanchi A, Dowdy DW, Davis JL, Worodria W, Yoo S, Joloba M, et.al.Sensitivity of direct versus concentrated sputum smear microscopy in HIV-infected patients suspected of having pulmonary tuberculosis. BMC Infect Dis. 2009, 9: 5310.1186/1471-2334-9-53.

12. Uddin M.K.M., Chowdhury M.R., Ahmed S, Rehman MT, Khatun R, Leth FV, et al. Comparison of direct versus concentrated smear microscopy in detection of pulmonary tuberculosis. BMC Res Notes 6, 291 (2013). 


\section{Table 1}

\begin{tabular}{|c|c|c|c|}
\hline \multicolumn{4}{|c|}{ Direct smear microscopy } \\
\hline & & Positive & Negative \\
\hline \multirow[t]{2}{*}{ Post decontamination smear microscopy } & Positive & 45 & 36 \\
\hline & Negative & 2 & 622 \\
\hline
\end{tabular}

\section{Table 2}

\begin{tabular}{lcc} 
& Direct smear microscopy & Post decontamination smear microscopy \\
Sensitivity & $36.84 \%$ & $60.52 \%$ \\
Specificity & $99.15 \%$ & $97.96 \%$ \\
PPV & $89.36 \%$ & $85.19 \%$ \\
NPV & $89.06 \%$ & $92.79 \%$ \\
\hline
\end{tabular}

